\title{
EMOGRAM: HELP (STUDENT) DESIGN RESEARCHERS UNDERSTANDING USER EMOTIONS IN PRODUCT DESIGN
}

\author{
Gerard VAN OS ${ }^{1}$ and Karin VAN BEURDEN ${ }^{2}$ \\ ${ }^{1}$ Saxion University of Applied Science, Industrial Design Department \\ 2Saxion UAS - Industrial Design Research Group
}

\begin{abstract}
Most experienced user researchers will recognise the following: When doing a user test, the things participants say during the interview not always match their facial expressions or how they acted during the test. They seem unable to explain what they, unconsciously, feel or think.

To counter this behaviour, we designed the Emogram that helps (student) design researchers capture (unconscious) emotional and intuitive responses of research participants with as little rationalisation by the participants as possible.

The work of Daniel Kahneman [1] is used to explain how the Emogram works cognitively. Kahneman showed that the human brain uses two systems to form thoughts: system 1, intuitive and fast, and system 2, rational and slow. System 1 reacts almost immediately while system 2 thinks things over and tries to rationalise the response of system 1 . The continues battle between these systems influences the response of research participants. Because of this, inexperienced design students can easily be put on the wrong foot as they are still learning how to read participant responses.

Not much is needed to do an Emogram: paper, pencil and some sticky notes will suffice. However, because participants sometimes feel that they don't have enough time to complete the Emogram, also a web version is being developed.

The tool is 'work in progress' and there are several issues that need further research. However, first experiences are that student researchers acquire more accurate insights about the participants unconscious thoughts in relation to product design.
\end{abstract}

Keywords: Emotion, unconscious, thoughts, rational, Kahneman, user-product-research

\section{INTRODUCTION}

To understand how the target users, think about a new product - or software or service -, many product designers visit target user representatives for tests and interviews. At some point, participants are asked to give words and meaning to their thoughts and feelings verbally in order to explaining them to the researcher. But the answers are often quite different from the nonverbal expressions or actions observed during the sessions. It is not clear why participants behave like this: Is it because they want to be friendly to the researcher? Do they want to answer with what they think the researcher wants to hear? Is it, maybe, hard or even impossible to find words for what they feel?

Several tools have been developed by user researchers aiming at getting an 'honest' answer from the user. The list of tools contains, amongst a lot of others, questionnaires like CSUQ (Computer System Usability Questionnaire) [1] and the Usefulness, Satisfaction, and Ease of Use questionnaire [2] [3]. A tool designed by David Travis [7] that offers users a randomised list of words from which they choose the ones that best match their thoughts. These tools address a problem that Israeli-American psychologist Daniel Kahneman had observed and explained by the following model: in the human brain, the intuitive and rational systems exist next to each other and these systems hardly interact with one another. The model explains rather well the inability of users to explain their thoughts, especially the emotional ones. 
In his book Thinking, Fast and Slow, Kahneman [4] describes two thought forming systems in the brain and how they interact. System 1 is what he calls the "fast, automatic, frequent, emotional, stereotypic, unconscious system"; system 2 is the "Slow, effortful, infrequent, logical, calculating, conscious system". In short: system 1 is the intuitive system; system 2 is the rational system. Also, as system 1 is autonomous and, because of its speed, efficient, it only requires little energy. System 2, on the other hand, requires quite some (attentional) energy but because of that it is able to explain, filter and correct the intuitive decisions of system 1. Obviously, both systems have their flaws: system 1 acts quickly but can make an error while doing so and system 2 takes time to think things through but will sometimes come to irrational results.

How than, does this apply to interviewing users? Let's assume that the researcher wants to find out how user-friendly a new product is. After defining the target user group, target user representatives are visited and asked to do some task with the product while talk-out-loud while doing so. This is quite a common user research approach. In terms of Kahneman's model: What the researcher sees happening between the user and the product is system 1 at work while the talk-out-loud part is system 2 output. However, the talking always lags the doing because system 2 takes much more time to explain the things system 1 is doing in a flash.

Product designers are, or should be, interested in the system 1 outcomes during a user test because these outcomes better reflect how users think and feel about the product (than the rational system 2 ones). In fact, most experienced product designers and researchers intuitively (!) do understand this. Design students, however, lack this experience and largely assume that what the participants tells them verbally is the truth. The result is that they come up with a redesign based on the wrong input. The redesign will, even when tested with the same participants, be unsuccessful. So, for student designers, a tool that taps into system 1 will be very helpful.

\section{THE TOOL}

What is needed is a tool that lets researchers learn about the users' real, not rationalised thoughts and insights. A tool that can be used during interviews and observations. A tool that gives way to system 1 as much as possible and without much interference from system 2 .

A paper from Sabrina Duda [5] describing the use of a mind map to create an association diagram about, in her case, how people feel about brands turned out to be very useful. A mind map, arguably invented by Buzan [6], in short, is a visualisation with the subject at the centre and with related associations branching from there. Mind map is a tool that is often used during product design to, e.g., visualise or collect/document different design ideas. The main differences between Dudas' map and the 'standard' mind map is that no line is drawn between associations and the subject and that there are no sub-branches springing from the associations. Contrary to mind maps, also, is that in Dudas' map the relative distance between associations and the centre subject relates to the importance or weight of the association. The closer an association is to the subject, the more important it is. Dudas' paper also suggested to compare diagrams before and after changing features of the centre subject in order to find out how that affect the associations.

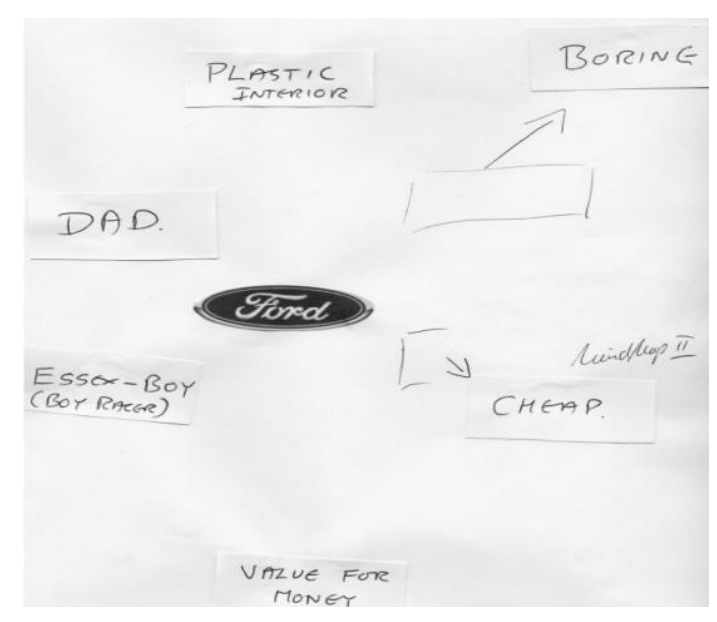

Figure 1. Association diagram taken from the paper of Sabrina Duda [5] 


\section{1 'Emogram'}

To facilitate our design students in getting and understanding the right response from users, we developed the Emogram based on the ideas of Dudas' mind map. We added concentric circles on a sheet of paper with the subject in the centre as shown in Error! Reference source not found.. The idea is that thoughts, likes, or doubts are written on sticky notes and put on the paper. More important notes should be positioned closer to the subject. Emogram is a contraction of the word's emotions and diagram. The circles were introduced to help users position different thoughts and to facilitate the analysis process, for instance in determining the distances between associations and with the main subject.

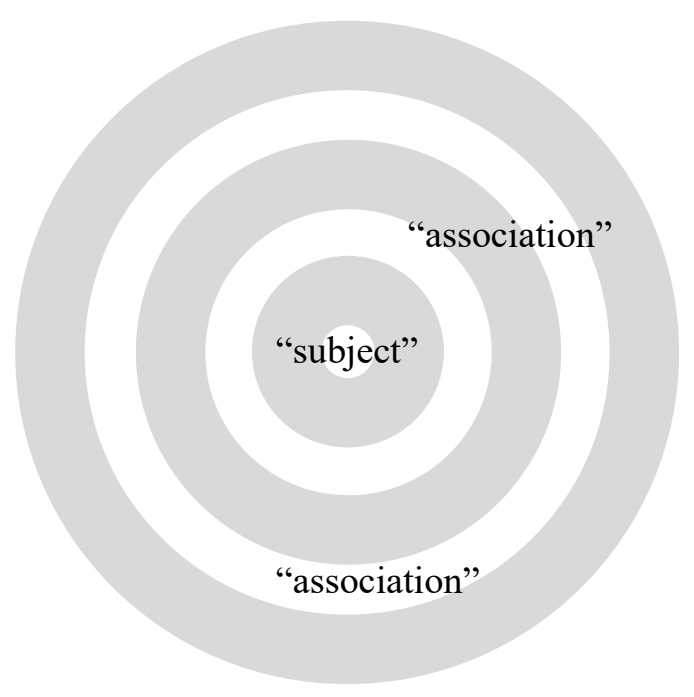

Figure 2. Area diagram used to position associations around a centre subject (own picture)

\subsection{How to use it}

User research is a very important part of product design. A major research area of the designers at the Research Group Industrial Design and the related Industrial Product Design course is to learn about and understand the real needs, perception, and attitudes of target user by involving these users and other stakeholders at an early stage in the design process. The Emogram turns out to be a very useful tool for this. After a brief introduction, participants are asked to fill in the paper Emogram. At the centre, a visual or the name of the examined product or product feature is placed. Participants are then asked to write thoughts, feelings, or doubts about the subject on sticky notes. The notes are put the Emogram paper in such a way that notes closer to the subject are more important than the ones further away. Participants are asked to do the exercise without much thinking and without talking. Only when participants are finished, they are asked to explain their notes. Every participant is asked to create an individual Emogram.

The tool can be used in different ways. It can, for instance, be used to record participants opinion about the examined product (feature) itself. But it can also be used to determine changes in user emotions and thoughts depending on a redesign of the product or product feature. Participants are then asked to create an initial Emogram based on the product as is, and then asked to create a new Emogram (or make changes to the first one) based on the redesign. Similarly, participants can be asked to create an Emogram for similar products of different manufacturers. Only when all Emograms are created, participants can be asked to explain the differences in the generated Emograms.

\subsection{Analysing diagrams}

To analyse the Emograms, a list of associations mentioned by the individual participants is made with the association closest to the subject at the top. Then the lists are compared, and an overall list is made. One of the problems is that participants can use different words for the same association! To counter this problem, it was decided to always have participants explain their words or notes after (!) they created the Emogram, even though this means that system 2 tries to make sense of system 1 doings. 
These explanations should help the researcher to understand and detect similar associations amongst participants. When comparing the results of diagrams before and after, e.g., a redesign, the designer can see how the redesign affected the associations and decide if the redesign is a good one.

\subsection{Experiences so far}

In 2012, the tool was used in a research project on artificial grass. Four buyers of artificial grass and four participants who didn't each created a diagram. (The non-buyers can be characterised as potential or future buyers.) The participants were asked to create a diagram about artificial grass before they were shown samples of the artificial grass. In the aftermath of the original study, an in-depth analysis of the generated diagrams was done after the study. Error! Reference source not found. shows the result of overlaying three Emograms created by buyers and with different sticky colours per buyer. The numbers in the circles were added during the analysis by the researchers to determine order and the relative positions of the associations. The analysis concluded that the tool has potential for researching experiences. The analysis also showed that the non-buyers generated more associations, but that the buyers had more positive ones.

As a result of this first trial, we determined that it may be useful to make a difference in positive and negative thoughts and emotions right from the start. Another assumed improvement was to add some ranking mechanism to indicate how strong some thought or emotion was. This improved version of the tool was then used during a workshop on 2014 World Usability Day at the Design Centre of the Twente University with some 20 participants and a workshop in 2015 with 30+ Industrial Design students at the University Twente. Error! Reference source not found. shows an overlay of several Emograms regarding an egg-timer, one of the products used during World Usability Day. This version of the Emogram had the subject at the far left and the positive and negative thoughts or feelings had separate area's in the diagram. Also, the vertical distance with the centre line should indicate the 'weight' of an association.

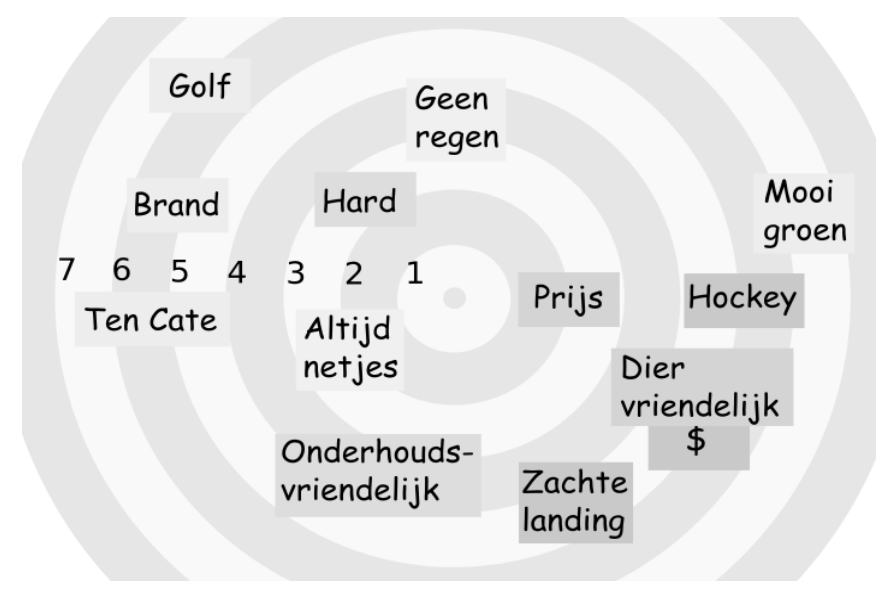

Figure 3. Overlaying Emograms of artificial grass study ('buyers', own picture)

Even though this change made sense to the designers/researchers, the trials with the improved version of the Emogram showed that participants had more difficulties generating the diagram. Although trials are still ongoing, it is quite likely that the reason for that is that participants had to think too much! Once a thought came into their mind, they not only had to decide whether it was a positive or negative thought, but the participants also had to decide how strong it was and where to put it in relation to the other thoughts. Participants were forced to use system 2 reasoning to give meaning to the system 1 thought. Because of this, we went back to the original layout and protocol of generating an Emogram. It usually takes 15-30 minutes to generate an Emogram, depending on the subject. Sometimes that is too long, especially when visiting participants at their workplace. For example: The tool was planned to be used in its original form in two research projects about anti-microbial coatings in hospitals and on clothing. After the first couple of interviews, it was clear that participants, busy nurses, didn't have the time to create diagrams. We decided to skip that part of the research and to discard the diagrams already generated. The participants that did create one, however, were positive about the Emogram. 


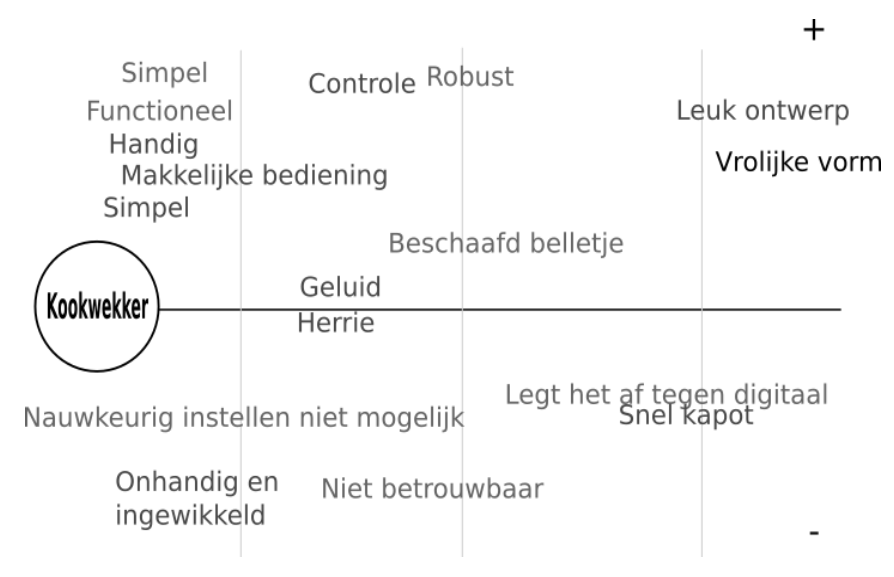

Figure 4. Emogram with areas for positive and negative thoughts and emotions (own picture)

\section{DISCUSSION, RECOMMENDATIONS}

As explained above, we have experimented with several layouts and protocols for generating Emograms. We asked participants to talk-out-loud only to discovered that they were again forced to rationalise their intuition, something that we tried to overcome. We redesigned the layout so that positive and negative associations were separated and that participants could give weight to their chosen words. Again, we found that participants had to rationalise their choices.

Today, we have returned to the original idea where participants positioning their thoughts without much thinking and talking. Only after they are finished, participants are asked to provide clarification of their diagram. However, as researchers we (have to) realise that this is a rationalisation of what they did.

What we also discovered was that time is a scarce commodity. Especially when visiting people at their workplace, we found that there is almost no time for doing something as intensive as creating an Emogram.

Another issue is the analysis of results: How to compare diagrams from different participants? It is not simply overlaying all diagrams as participants use different distances and different words to otherwise similar thoughts. In fact, all distances need to be normalised (recalculated) in order to be able to compare them over different diagrams.

To counter two of the issues, the time it takes to generate a diagram and facilitating analysis and comparison of generated diagrams, a web version of the Emogram tool is being worked on. It is still at the early stages of development, but this software version will be tested and further developed with Industrial Design students, students taking a class on qualitative research and during research projects of the Industrial Design Research Group.

The research protocol for this web version is that participants are invited to generate words or phrases for their thoughts and emotions towards the subject. They can create their own words/phrases or use words/phrases that have already been entered before by prior participants or by the researchers. Next, participants are presented their list of words/phrases where the first word/phrase that they entered is at the top of the list. The assumption is that the first word or phrase that they generated is the most important one. Participants are allowed to change the order of their list. Finally, the list is saved, and the researcher is offered the opportunity to compare the generated lists of all participants. A major concern with this textual implementation is that it is quite different from the original paper-and-pencil one. In the web-version, participants can only create a list of words and phrases and order them but, for now, cannot change the distance between them. Also, in the present web version it is not possible to have associations of similar importance. The assumption is that the web-tool will evolve over time to overcome this limitation. 


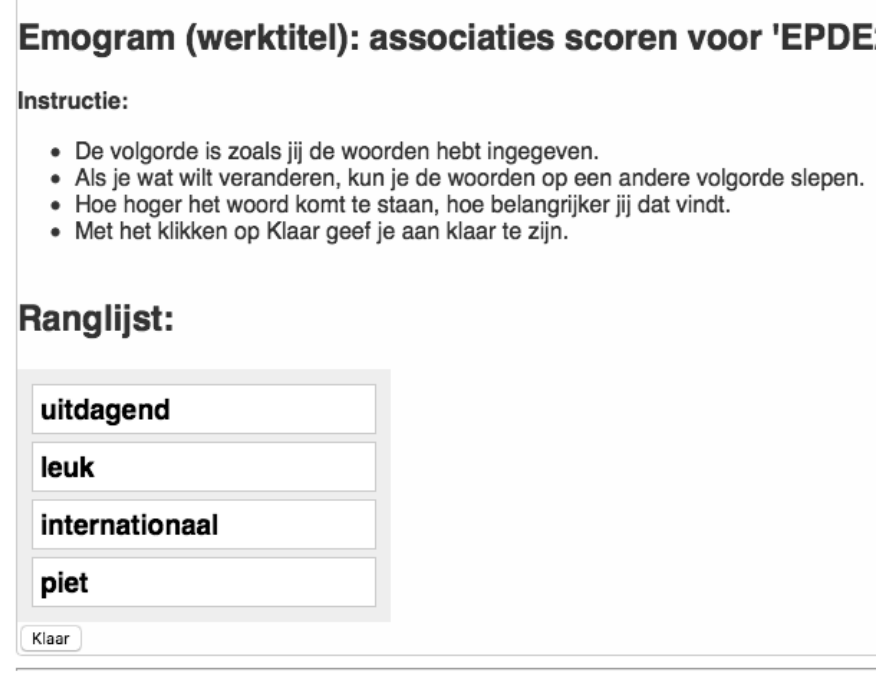

Figure 5. Screenshot of web version of Emogram (own picture)

Even though the paper version is used by several students, the main focus our research group was on how the tool could help us understanding the emotions of participants. We are thinking about a way to have the tool actually help and develop the understanding of user emotions by students. We could, e.g., have students write down their thoughts and observations about the participant in relation to the subject based on what they saw and heard during the session and have them compare and evaluate these notes when they are interpreting the generated Emograms.

Over the next couple of months, students participating in a class on User Research Methods will use both the web version and the paper-and-pencil version of the Emogram during two assignments. They are asked to evaluate their experiences with the tool and have to come up with recommendations how to go on.

\section{REFERENCES}

[1] Perlman G. Computer System Usability Questionnaire. Available: https://garyperlman.com/quest/quest.cgi?form=CSUQ [Accesses on 2019, 26 February]

[2] Perlman G. USE Questionnaire: Usefulness, Satisfaction, and Ease of use. Available: https://garyperlman.com/quest/quest.cgi?form=USE [Accessed on 2019, 26 February]

[3] Lund A. Measuring Usability with USE questionnaire. Available: https://www.researchgate.net/publication/230786746_Measuring_Usability_with_the_USE_Ques tionnaire\&usg=AOvVaw1_c8Xk2ejNgnjli9R_UYaE [Accessed 2019, 26 February]

[4] Kahneman D. Thinking, Fast and Slow, 2011 (Farrar, Strauss and Giroux, United States).

[5] Duda S. Mind Maps: An evaluation Method of Web User Brand Experience. Available: http://www.smiling.club/assets/fileupload/mind_map_duda_e.pdf [Accessed on 2019, 27 February]

[6] Buzan T. The Mind Map Book., 1991 (Penguin, New York, USA).

[7] Travis D. Measuring satisfaction: Beyond the usability questionnaire. Available: https://www.userfocus.co.uk/articles/satisfaction.html [Accessed 2019, 5 March] 